\title{
Pairing of Fermions with Arbitrary Spin
}

\author{
${ }^{*+}$ Tin-Lun Ho and ${ }^{*}$ Sungkit Yip \\ *Physics Department, The Ohio State University, Columbus, OH 43210 \\ + Institute for Theoretical Physics, University of California at Santa Barbara, CA 93106
}

\begin{abstract}
Motivated by the recent success of optical trapping of alkali Bose condensate, we have studied the superfluid state of optically trapped alkali fermions, which can have Cooper pairs with total spin $J \geq 2$. In this paper, we shall discuss the general structure of these large spin Cooper pairs and their close relation with singlet Cooper pairs with non-zero orbital angular momentum. We also present the exact solution for the $J=2$ pairing which shows a surprising change of ground state as the spin $f$ of the constituent fermion increases.
\end{abstract}

The discovery of Bose-Einstein condensation [1] in atomic gases has stimulated many new research directions. Among these is the search of the superfluid phases of alkali fermions. This search has become even more exciting in view of the recent success of confining Bose condensates in optical traps [2]. Since optical traps are non-magnetic, the spin of the trapped atoms are no longer frozen as they were in magnetic traps. This leads to a new class of superfluid phenomena. In the case of spin-1 Bose gas like ${ }^{23} \mathrm{Na}$ and ${ }^{87} \mathrm{Rb}$, one of us [3] has recently pointed out ${ }^{23} \mathrm{Na}$ and ${ }^{87} \mathrm{Rb}$ should have a non-magnetic and ferromagnetic spinor condensate respectively, according to the current estimates of their scattering lengths 四. Very recently, experiments at MIT [5] have verified the basically non-magnetic spinor nature of ${ }^{23} \mathrm{Na}$ and found that its magnetic interaction is indeed antiferromagnetic [3].

The physics of alkali fermions in optical traps is equally rich. The fact that all alkali fermions (except ${ }^{6} \mathrm{Li}$ ) have hyperfine spins (or simply "spins") $f>1 / 2$ in their lowest hyperfine manifold implies that their Cooper pairs can have total spins $J>1$. Fermions 
like ${ }^{22} \mathrm{Na}$ and ${ }^{134} \mathrm{Cs}$ which have $f=5 / 2$ and $7 / 2$ can have Cooper pairs with total spin as high as 4 and 6 . From the example of superfluid ${ }^{3} \mathrm{He}$, one can be sure that the internal structure of these large spin Cooper pairs will generate a multitude of macroscopic quantum phenomena. The purpose of this paper is to point out the structure these large spin Cooper pairs, and a surprising change in behavior of a spin- $J$ Cooper pair as a function of fermion $\operatorname{spin} f$.

As a first step, we shall focus on homogenous dilute Fermi gases in zero magnetic fields. It is important to understand the homogeneous situation before studying the trapped cases [6]. Moreover, the physics of homogeneous systems are important in their own right. At first sight, the weak field limit seems difficult to achieve, for even the Earth magnetic field amounts to $10^{-4} \mathrm{~K}$, enough to polarize the whole gas. Despite this "strong" background field, which can be shielded off to a large extent, one can reduce it effectively to the weak field limit by specifying the total spin $S$ of the system. Since the dynamics of these systems is spin conserving [3], a prepared spin $S$ out of equilibrium with an external field cannot relax to its equilibrium value. The system therefore sees an effective field which would have been in equilibrium with the prepared $S$. By choosing $S$ appropriately, the effective field can be made much smaller than the external one. This method has very recently been used by Ketterle's group to study the spinor nature of the ${ }^{23} \mathrm{Na}$ condensate [5].

Of course, for a pairing state to be observable, its pairing interaction has to be sufficiently negative to produce an observable $T_{c}$. While the scattering lengths of some alkali fermions have been calculated, they remain unknown for many alkalis. (See later discussion). In view of the lack of information, we have performed a general study of the large spin Cooper pairs. In particular, we shall discuss the $J=2$ pairing in detail. This is the simplest among all large spin pairing which also has an exact solution. The phenomena contained in this case reveal the rich physics installed, which turns out to be remarkable indeed. For simplicity, we shall call the S-wave spin- $J$ Cooper pairs (made up of two spin $f$ fermions) "spin" Cooper pairs, and singlet Cooper pairs with orbital angular momentum $J$ (made up of two spin-1/2 fermions) "orbital" Cooper pairs. Let us first summarize our findings : 
(A) The structure of "spin" Cooper pairs is analogous to that of "orbital" Cooper pairs with the same angular momentum. This allows one to obtain information of the former from the latter, for which an exact solution already exists for $J=2$.

(B) The structure of a spin- $J$ Cooper changes as the spin $f$ of the constituent fermions increases beyond a critical value. For Cooper pairs with spin $J=2$, they are "ferromagnetic" (or "axial") if $f \geq 7 / 2$, but non-magnetic (or "real") if $f \leq 5 / 2$. This change of character as a function of $f$ is a result of maximizing the phase space for pairing and is independent of interaction parameters, as long as they favor $J=2$ pairing.

Free energy: The low energy effective Hamiltonian of a spin- $f$ dilute Fermi gas with s-wave interactions has been derived in ref. [3]. It is rotationally invariant in spin space, and is of the form $H-\mu N=\int \mathrm{d} \mathbf{x} \psi_{\alpha}^{+}(\mathbf{x}) \mathcal{H}_{\alpha \beta}^{o}(\mathbf{x}) \psi_{\beta}(\mathbf{x})+\frac{1}{2} \int \mathrm{d} \mathbf{x} \psi_{\alpha}^{+}(\mathbf{x}) \psi_{\beta}^{+}(\mathbf{x}) \Gamma_{\alpha \beta ; \mu \nu} \psi_{\mu}(\mathbf{x}) \psi_{\nu}(\mathbf{x})$, $\mathcal{H}_{\alpha \beta}^{o}(\mathbf{x})=-\frac{\hbar^{2}}{2 M} \nabla^{2} \delta_{\alpha \beta}-\gamma \mathbf{B} \cdot \mathbf{F}_{\alpha \beta}$,

$$
\Gamma_{\alpha \beta ; \mu \nu}=\sum_{F=0}^{2 f-1} g_{F} \sum_{m=-F}^{F}<f f \alpha \beta|f f ; F m><f f ; F m| f f \mu \nu>
$$

where $M$ is the mass of the fermion, $\langle f f ; F m| f f \mu \nu>$ is the Clebsch-Gordan coefficient for forming a total spin $F$ from two spin- $f$ particles, $g_{F}=4 \pi \hbar^{2} a_{F} / M$, and $a_{F}$ is the s-wave scattering length of two spin- $f$ fermions in the scattering channel with total spin $F$. Because of antisymmetry of the fermions, only even $F$ 's appear in eq.(1).

The order parameter of an S-wave superfluids is $\Psi_{\alpha \beta}(\mathbf{x})=<\psi_{\alpha}(\mathbf{x}) \psi_{\beta}(\mathbf{x})>$, which is a $(2 f+1) \times(2 f+1)$ antisymmetric matrix in spin space. For homogeneous systems, $\Psi_{\alpha \beta}$ is independent of $\mathbf{x}$. It is convenient to define the gap function

$$
\Delta_{\alpha \beta}=\Gamma_{\alpha \beta ; \mu \nu} \Psi_{\mu \nu}
$$

The free energy according BCS theory is

$$
\mathcal{F}=\frac{1}{2} \operatorname{Tr} \Delta^{+} \Gamma^{-1} \Delta-\frac{k_{B} T}{2} \sum_{\mathbf{k} \omega_{n}} \sum_{\ell=1}^{\infty} \frac{1}{\ell} \operatorname{Tr}\left[\Delta \tilde{G}\left(\mathbf{k} \omega_{n}\right) \Delta^{+} G\left(\mathbf{k} \omega_{n}\right)\right]^{\ell}
$$

where $\Gamma^{-1}$ is given by eq.(1]) with $g_{F}$ replaced by $g_{F}^{-1}, \omega_{n}=(2 n+1) \pi k_{B} T$ are the Matsubara frequencies, $G_{\alpha \beta}\left(\mathbf{k}, \omega_{n}\right)$ and $\tilde{G}_{\alpha \beta}\left(\mathbf{k}, \omega_{n}\right)$ are normal Greens functions satisfying matrix equation $\left(i \omega_{n}-\mathcal{H}_{o}(\mathbf{k})\right) G\left(\mathbf{k} \omega_{n}\right)=1,\left(i \omega_{n}+\mathcal{H}_{o}^{T}(\mathbf{k})\right) \tilde{G}\left(\mathbf{k} \omega_{n}\right)=1$. 
General structure of Cooper pairs with spin angular momentum $J$ : Under a spin rotation $U=\exp (-i \vec{\theta} \cdot \mathbf{F}), \psi_{\alpha} \rightarrow(U \psi)_{\alpha}$. This implies $\Psi \rightarrow \Psi^{\prime}=U \Delta U^{T}$, and hence $\Delta \rightarrow \Delta^{\prime}=$ $U \Delta U^{T}$. For gap functions that transform like an angular momentum state $\mid J m>$, they must satisfy

$$
\left[U \Delta_{m}^{(J)} U^{T}\right]_{\alpha \beta}=D_{m m^{\prime}}^{(J)}(\vec{\theta})\left[\Delta_{m^{\prime}}^{(J)}\right]_{\alpha \beta}
$$

It is easy to see that the solution of eq. (乘) is $\left(\Delta_{m}^{(J)}\right)_{\alpha \beta} \propto<f f \alpha \beta \mid f f ; J m>$. The general structure of the spin- $J$ gap function is therefore

$$
\left(\Delta^{(J)}\right)_{\alpha \beta} \propto \sum_{m=-J}^{J} c_{m}<f f \alpha \beta \mid f f ; J m>, \quad \text { or } \quad\left|\Delta^{(J)}>\propto \sum_{m=-J}^{J} c_{m}\right| J m>
$$

where the second expression in eq.(5) is simply the first written in abstract form.

To find the $\Delta^{(J)}$ that minimizes the energy, and to illustrate the relation of "spin" and "orbital" Cooper pairs, it is useful to consider a different representation of $\Delta^{(J)}$. First, we note that the singlet state $\left(\Delta^{(0)}\right)_{\alpha \beta} \propto \eta \equiv<f f ; 00 \mid f f \alpha \beta>\sqrt{2 f+1}$ satisfies $U \eta U^{T}=\eta$, and has the properties $\eta^{+} \eta=1, U^{+} \eta=\eta U^{T}$, and $F_{i} \eta=-\eta F_{i}^{T}$. Defining $\Delta \equiv \Xi \eta$, Eq.(四) then becomes $U \Xi_{m}^{(J)} U^{+}=D_{m m^{\prime}}^{(J)} \Xi_{m^{\prime}}^{(J)}$, which has the solution $\left[\Xi_{m}^{(J)}\right]_{\alpha \beta} \propto\left[Y_{J m}(\mathbf{F})\right]_{\alpha \beta}$, where $Y_{J m}(\mathbf{F})$ is a matrix obtained by first writing the spherical harmonic $k^{J} Y_{m}^{(J)}(\hat{\mathbf{k}})$ in a symmetric rectangular form, and then by replacing $k_{i}$ by the matrix $F_{i}[7]$. For example, since $k^{2} Y_{21}(\hat{\mathbf{k}}) \propto k_{z}\left(k_{x}+i k_{y}\right)$, we have $Y_{21}(\hat{\mathbf{F}}) \propto F_{z}\left(F_{x}+i F_{y}\right)+\left(F_{x}+i F_{y}\right) F_{z}$. The general form of the order parameter within the angular momentum $J$ subspace is then

$$
\Delta_{\alpha \beta}^{(J)}=\sum_{m=-J}^{J} c_{m}\left[Y_{J m}(\mathbf{F}) \eta\right]_{\alpha \beta}
$$

Using Wigner-Eckart theorem, it is easily seen that the two representation eq.(5) and eq.(6) are identical.

Next, we note that $r^{J} Y_{J m}(\hat{\mathbf{r}})$ is a homogenous polynomial of $\mathbf{r}$ satisfying Laplace's equation. It can therefore be written as $r^{J} Y_{J m}(\hat{\mathbf{r}})=A_{i_{1} i_{2} \ldots i_{J}} r_{i_{1}} r_{i_{2}} \ldots r_{i_{J}}$, where $A_{i_{1} i_{2} \ldots i_{J}}$ is symmetric in all its indices and vanishes whenever any two indices contract. We can then write $\Delta^{(J)}$ as 


$$
\Delta^{(J)}=\sum_{i_{1} \ldots i_{J}} A_{i_{1} i_{2} \ldots i_{J}} F_{i_{1}} F_{i_{2}} \ldots F_{i_{J}} \eta
$$

It is also useful to compare the spin structure of $\Delta^{(J)}$ in eq.(7) with the orbital structure of the singlet Cooper pairs of spin-1/2 fermions. The order parameter of the latter is $\Delta(\mathbf{k})=<c_{\uparrow}(\mathbf{k}) c_{\downarrow}(-\mathbf{k})>$, where $c_{\uparrow}^{+}(\mathbf{k})$ creates a spin $+1 / 2$ fermion with momentum $\mathbf{k}$ at the Fermi surface. For pairing with even orbital angular momentum $J, \Delta^{(J)}(\mathbf{k})=\sum_{m} c_{m} Y_{J m}(\hat{\mathbf{k}})$, or

$$
\Delta^{(J)}(\mathbf{k})=\sum_{i_{1} \ldots i_{J}} A_{i_{1} i_{2} \ldots i_{J}} k_{i_{1}} k_{i_{2}} \ldots k_{i_{J}}
$$

Comparing eq.(7) and eq.(8), one finds that they are almost identical except that the $F_{i}$ 's are non-commuting matrices whereas the $k_{i}$ s are c-numbers. On the other hand, this means that these two structures approach each other as $f$ increases, since the spin operator $\mathbf{F}$ behaves more like a classical vector.

The general scheme for determining $\Delta^{J}$ and the $J=2$ pairing : At temperature is lowered, superfluid condensation first takes place at the (even) angular momentum channel $J$ with largest negative coupling constant $g_{J}$. The free energy eq.(3) to the quartic order in $\Delta^{(J)}$ is

$$
\mathcal{F}=-\frac{1}{2} \alpha \operatorname{Tr} \Delta^{(J)+} \Delta^{(J)}+\frac{1}{4} \beta \operatorname{Tr}\left(\Delta^{(J)} \Delta^{(J)+}\right)^{2}
$$

where $\alpha=N(0) \ln \left(T_{c} / T\right), T_{c}=1.14 \epsilon_{F} e^{-1 /\left(\left|g_{J}\right| N(0)\right)}=1.14 \epsilon_{F} e^{-\pi /\left(2 k_{F}\left|a_{J}\right|\right)}, N(0)$ is the density of state at the fermi surface per spin, $\beta=7 \zeta(3) /\left(8 \pi^{2} T_{c}^{2}\right)$, and $\epsilon_{F}$ and $k_{F}$ are the Fermi energy and momentum. To determine $\Delta^{(J)}$, we substitute eq.(7) into eq.(9) and find the matrix $A$ that minimizes the energy. In the following, we shall present the exact solution for for S-wave $J=2$ Cooper pairs formed by spin- $f$ fermions. The solutions of $J>2$ Cooper pairs will be studied elsewhere for they require much lengthier calculations than the $J=2$ case, which is already lengthy. Our method, however, applies to all $J \geq 2$ pairs.

¿From eq.(9), one can see that $\mathcal{F}$ is of the form

$$
\mathcal{F}=-\frac{\alpha}{2} A_{i j} A_{p q}^{*} \operatorname{Tr}\left(F_{i} F_{j} F_{p} F_{q}\right)+\frac{\beta}{4} A_{i j} A_{k \ell}^{*} A_{p q} A_{s t}^{*} \operatorname{Tr}\left(F_{i} F_{j} F_{k} F_{\ell} F_{p} F_{q} F_{s} F_{t}\right)
$$


After evaluating the traces (see Appendix), we find

$$
\begin{gathered}
\mathcal{F}=-\tilde{\alpha} \operatorname{Tr} A A^{+}+\beta_{1}\left|\operatorname{Tr} A^{2}\right|^{2}+\beta_{2}\left(\operatorname{Tr} A^{*} A\right)^{2}+\beta_{3} \operatorname{Tr}\left(A^{2} A^{* 2}\right) \\
\beta_{1}=\frac{\beta}{4}\left[-\frac{29}{70} I_{2}+\frac{121}{60} I_{4}-\frac{22}{15} I_{6}+\frac{4}{35} I_{8}\right] \\
\beta_{2}=\frac{\beta}{4}\left[-\frac{2}{70} I_{2}+\frac{1}{30} I_{4}+\frac{4}{15} I_{6}+\frac{8}{35} I_{8}\right] \\
\beta_{3}=\frac{\beta}{4}\left[\frac{3}{5} I_{2}-\frac{8}{3} I_{4}+\frac{16}{15} I_{6}\right]
\end{gathered}
$$

where $I_{n} \equiv \sum_{m=-f}^{f} m^{n}$, and $\tilde{\alpha}=\frac{\alpha}{12}\left[4 I_{4}-I_{2}\right]$.

Eq.(11) is identical to the free energy of a general $d$-wave singlet superfluids The minimization problem of eq.(11) was solved by Mermin [8]. Only three equilibrium phases are possible [9]:

(I) "Axial" state : When $\beta_{3}>-\beta_{1}+\left|\beta_{1}\right|,, \Delta \propto Y_{22}(\mathbf{F}) \eta$,

(II) "Cyclic" state : When $0>\beta_{3}>-6 \beta_{1}, \Delta \propto\left(F_{x}^{2}+e^{2 \pi i / 3} F_{y}^{2}+e^{4 \pi i / 3} F_{z}^{2}\right) \eta$,

(III) "Real" state : When $\beta_{3}<-4 \beta_{1}-2\left|\beta_{1}\right|, \Delta \propto\left[\zeta_{1} Y_{20}(\mathbf{F})+\zeta_{2}\left(Y_{22}(\mathbf{F})+Y_{2,-2}(\mathbf{F})\right)\right] \eta$, where $\zeta_{1}$ and $\zeta_{2}$ are real.

The portion of the phase diagram in $\beta_{1}-\beta_{3}$ space relevant for our discussion is shown in fig.1. Using eq.(12) to eq.(14), we note that $\left(\beta_{1}, \beta_{3}\right)$ is in region III for $f=\frac{3}{2}$ and $\frac{5}{2}$, and in region I for $f \geq \frac{7}{2}$. The superfluid is therefore a "real" state for $f=\frac{3}{2}$ and $\frac{5}{2}$, but changes to the "axial" state for $f \geq \frac{7}{2}$.

This change of pairing behavior can be understood as follows. As mentioned before, as $f \rightarrow \infty$, the order parameters in eq.(17) and eq.(8) become identical, and that the energy eq.(9) becomes that the weak coupling d-wave superfluid, which has an optimum order parameter $Y_{22}(\hat{\mathbf{k}})[8]$. (This state has "more pairing" $Y_{20}$ and $Y_{2 \pm 1}$ in the sense that its absolute square only has point nodes whereas both $\left|Y_{20}(\hat{\mathbf{k}})\right|^{2}$ and $\left|Y_{2, \pm 1}(\hat{\mathbf{k}})\right|^{2}$ have line nodes). On the other hand, in the most quantum case $f=\frac{3}{2}$, there are four degenerate Fermi surfaces, labelled by $m_{z}= \pm \frac{3}{2}, \pm \frac{1}{2}$. The structure of the axial 
and the real state are given by $\left|\Delta_{\text {axial }}>=\right| 2 ; 2>=\frac{1}{\sqrt{2}}\left(\left|\frac{3}{2}, \frac{1}{2}>-\right| \frac{3}{2}, \frac{1}{2}>\right), \mid \Delta_{\text {real }}>=$ $\zeta_{1} \mid 2 ; 0>\quad+\zeta_{2}(|2 ; 2>+| 2,-2>)=\quad \frac{\zeta_{1}}{2}\left[\left(\left|\frac{3}{2}, \frac{-3}{2}>-\right| \frac{-3}{2}, \frac{3}{2}>\right)+\left(\left|\frac{1}{2}, \frac{-1}{2}>-\right| \frac{-1}{2}, \frac{1}{2}>\right)\right]$ $+\frac{\zeta_{2}}{2}\left[\left(\left|\frac{3}{2}, \frac{1}{2}>-\right| \frac{1}{2}, \frac{3}{2}>\right)+\left(\left|\frac{-1}{2}, \frac{-3}{2}>-\right| \frac{-3}{2}, \frac{-1}{2}>\right)\right]$, where the state vectors with integer entries such as $\mid 2 ; 0>$ means $\mid \frac{3}{2} \frac{3}{2} ; J=2, m=0>$, those with half integer entries such as $\mid \frac{1}{2}, \frac{-1}{2}>$ means $\left|f=\frac{3}{2}, m=\frac{1}{2}>\right| f=\frac{3}{2} ; m=\frac{-1}{2}>$. One can see that the only two Fermi surfaces $\left(m=\frac{3}{2}\right.$ and $\left.\frac{1}{2}\right)$ are involved in the pairing in axial state, whereas all four Fermi surfaces are involved in the pairing of the real state. Since the real state maximizes the amount of pairing, it is favored in this extreme quantum case. As $f$ increases, the number of Fermi surfaces appearing in the axial state (i.e the spin state $\mid J=2, m=2>$ ) quickly increases. By the time when $f$ reaches $\frac{7}{2}$, the real state no longer has the advantage of involving most Fermi surfaces, and the system switches to the axial state, where the spin operator $\mathbf{F}$ begins to resemble a classical vector. We have thus established Statements (A) and $(\mathbf{B})$.

Observability: The long lived alkali fermions which have $f>\frac{1}{2}$ in their lowest hyperfine manifold are ${ }^{22} \mathrm{Na},{ }^{40} \mathrm{~K},{ }^{86} \mathrm{Rb},{ }^{132} \mathrm{Cs},{ }^{134} \mathrm{Cs}$, and ${ }^{136} \mathrm{Cs}$, which have $(f=$ $5 / 2,9 / 2,5 / 2,3 / 2,7 / 2,9 / 2)$ and lifetimes (2.5 yrs, $10^{9}$ yrs, 18 days, 6 days, 2 yrs, 13 days) respectively [10]. According to the recent calculation of Greene, Burke, and Bohn [4], the scattering lengths of ${ }^{40} \mathrm{~K}$ are positive, hence unfavorable for pairing. At present, there is no information about the scattering lengths of the Cs fermions. On the other hand, $a_{4}=-65(+40,-120) a_{B}, a_{2}=-130(+40,-70) a_{B}, a_{0}=-145(+40,-65) a_{B}$ for ${ }^{86} \mathrm{Rb}$; and $a_{4}=-108(+27,-40) a_{B}, a_{2}=-115(+32,-50) a_{B}, a_{0}=-117(+34,-55) a_{B}$ for ${ }^{22} \mathrm{Na}$, where $a_{B}$ is the Bohr radius and the numbers in the bracket are error bars.

To estimate $T_{c}$. we use the value of $k_{F}$ and $\epsilon_{F}$ at the center of the trap. For an anisotropic trap with frequencies $\omega_{\perp}$ and $\omega_{z}$ in the $x y$-plane and along $z$, it is easy to show that $k_{F} a_{2}=$ $\left(\frac{R}{a_{\perp}}\right)\left(\frac{a_{2}}{a_{\perp}}\right), \epsilon_{F}=\frac{1}{2} \hbar \omega_{\perp}\left(\frac{R}{a_{\perp}}\right)^{2}$, where $a_{\perp}=\sqrt{\hbar / M \omega_{\perp}}$, where $R$ is the radius of the cloud in the $x y$-plane related to the total number of particles $N$ as $\frac{R}{a_{\perp}}=\left(\frac{48 N \lambda}{(2 f+1)}\right)^{1 / 6}$, with $\lambda \equiv \omega_{z} / \omega_{\perp}$. For an isotropic trap $(\lambda=1)$ with $\omega_{\perp} / 2 \pi=2000 \mathrm{~Hz}$, the expression $T_{c}(J=2)=1.14 \epsilon_{F} e^{-\pi / 2 k_{F}\left|a_{2}\right|}$ 
gives $T_{c}(J=2) \sim 1.9 \times 10^{-8} \mathrm{~K}$ for ${ }^{22} \mathrm{Na}$ with $N=4 \times 10^{6}$ atoms and $T_{c}(J=2) \sim 2.3 \times 10^{-7} \mathrm{~K}$ for ${ }^{86} \mathrm{Rb}$ with $N=10^{6}$ atoms. Since the lowest temperature reached in current BEC experiments is $10^{-9} \mathrm{~K}$, these transition temperatures of fermions (which can be made higher by increasing the trap frequency or the anisotropy $\lambda$ ) appear to be feasible. Since $g_{0}$ is most negative, singlet instead of $J=2$ pairing will first occur in zero field. This, however, does not mean that all higher spin pairing states are non-observable. The singlet spin states can be efficiently suppressed in a magnetic field (obtained by specifying the total spin of the system as mentioned in the introduction), thereby revealing all other higher spin pairing states [11]. For length reasons, the effects of spin constraints will be discussed elsewhere.

Final Remarks: We have shown that the superfluid phenomena of alkali fermions become amazingly rich once the spin degrees of freedom are released. Should the current efforts of cooling alkali fermions to degenerate limit be successful, transferring the degenerate gas into an optical trap [2] will help one to uncover the superfluid phases discussed here. Since ${ }^{132} \mathrm{Cs}$, ${ }^{134} \mathrm{Cs}$, and ${ }^{136} \mathrm{Cs}$ have $f=3 / 2,7 / 2$, and $9 / 2$ in their lowest hyperfine multiplet respectively, if their scattering lengths turned out to be negative, our result predicts that like ${ }^{22} \mathrm{Na}$ and ${ }^{86} \mathrm{Rb}$, the ground state of ${ }^{132} \mathrm{Cs}$ will a "real" state, whereas ${ }^{134} \mathrm{Cs}$, and ${ }^{136} \mathrm{Cs}$ will be an "axial" state.

Appendix: Evaluation of the quartic term in eq.(10) : Denoting $\mathcal{I}_{4}=$ $A_{i j} A_{k \ell}^{*} A_{p q} A_{s t}^{*} \operatorname{Tr}\left(F_{i} F_{j} F_{k} F_{\ell} F_{p} F_{q} F_{s} F_{t}\right)$, we note that $\mathcal{I}_{4}=\mathcal{D}[\operatorname{Tr} U]_{o}$, where $\mathcal{D} \equiv$ $A_{i j} A_{k \ell}^{*} A_{p q} A_{s t}^{*} \frac{\partial^{2}}{\partial a_{i} \partial a_{j}} \frac{\partial^{2}}{\partial b_{k} \partial b_{\ell}} \frac{\partial^{2}}{\partial c_{p} \partial c_{q}} \frac{\partial^{2}}{\partial d_{s} \partial d_{t}}, U \equiv e^{-i \mathbf{a} \cdot \mathbf{F}} e^{-i \mathbf{b} \cdot \mathbf{F}} e^{-i \mathbf{c} \cdot \mathbf{F}} e^{-i \mathbf{d} \cdot \mathbf{F}} \equiv e^{-i \vec{\theta} \cdot \mathbf{F}}$, and the subscript "o" means $\mathbf{a}=\mathbf{b}=\mathbf{c}=\mathbf{d}=0$. Expanding $U$ in powers of $\vec{\theta}$, it is easy to see that $\mathcal{I}_{4}=$ $\sum_{n=1}^{4} \frac{(-1)^{n}}{(2 n) !} I_{2 n}\left(\mathcal{D} \theta^{2 n}\right)_{o}$, where $I_{2 n}=\sum_{m=-f}^{f} m^{2 n}$. Next, we note that the relation between $\theta$ and $\mathbf{a}, . ., \mathbf{d}$ is independent of $f$. For spin $1 / 2$ systems, the quantity $Q \equiv \operatorname{Tr}\left[e^{-i \mathbf{a} \cdot \vec{\sigma} / 2} e^{-i \mathbf{b} \cdot \vec{\sigma} / 2}\right.$ $\left.e^{-i \mathbf{c} \cdot \vec{\sigma} / 2} e^{-i \mathbf{d} \cdot \vec{\sigma} / 2}\right] / \operatorname{Tr}(1)=<e^{-i \vec{\theta} \cdot \vec{\sigma} / 2}>$ can be written as $\xi=Q-1=\sum_{n=1,2, . .} \frac{(-1)^{n}}{2^{2 n}(2 n) !} \theta^{2 n}$. Inverting this relation, we obtained $\theta^{2}$ as a power series of $\xi$, or $Q$. From this expression, we can calculate $\left(\mathcal{D} \theta^{2 n}\right)_{o}$ for $n=2$ to 4 by calculating $\mathcal{D} Q^{p}$ for $p=1,2,3,4$. The latter can be easily calculated because they involve only spin $1 / 2$ quantities. Evaluating $\mathcal{I}_{4}$ this way gives eq.(12) to eq.(14). 
Acknowledgement : TLH would like to thank Jim Burke for the estimates of the scattering lengths. This work is supported by a Grant from NASA NAG8-1441, and NSF Grants DMR-9705295 and DMR-9807284. 


\section{REFERENCES}

[1] M.H. Anderson, J.R. Ensher, M.R. Mathews, C.E. Weiman, and E.A. Cornell, Science 269, 198 (1995). K. B. Davis, M.-O. Mewes, M.R. Andrews, N.J. van Druten, D.S. Durfee, D.M. Kurn, and W. Ketterle, Phys. Rev. Lett. 75, 3969 (1995).

[2] D. Stamper-Kurn. M.R. Andrews, A.P. Chikkatur, S. Inouye, H.J. Miesner, J. Stenger, and W. Ketterle. Phys.Rev. Lett.80, 2027 (1998).

[3] T.L. Ho, Phys. Rev. Lett. 81, 742, (1998).

[4] J. Burke, C. Greene, and J. Bohn, to be published.

[5] J. Stenger, et.al. Spin domains in ground state spinor Bose-Einstein condensates, preprint.

[6] For not too small number of particles, the local fermi wavelength will be much less than the size of the cloud almost everywhere. One can therefore think of the order parameter locally. The proximity effect will however lower the $T_{c}$ somewhat from the estimate discussed below.

[7] G. Baym, Ch.17, Lectures on Quantum Mechanics, Benjamin/Cummings, 1969.

[8] N.D. Mermin, Phys. Rev. A 9, 868 (1974).

[9] The phases listed consist of degenerate manifolds. A general state is obtained from those listed by rotation and gauge transformations.

[10] Nuclear Wallet Cards, 5th ed., edited by Jagdish K. Tuli, Brookhaven National Laboratory.

[11] More precisely, the $J=0$ transition will be more strongly suppressed than the axial state $\Delta \propto Y_{22}(\mathbf{F}) \eta$ or the real state $\Delta \propto\left[Y_{22}(\mathbf{F})+Y_{2-2}(\mathbf{F})\right] \eta$.

Figure caption: Distribution of stable phases in the $\left(\beta_{1} / \beta_{2}, \beta_{3} / \beta_{2}\right)$ space for $J=2$ pairing 
88. Regions I, II, III are the stable regions of the "axial", "cyclic", and "real" states resp. $\mathbf{U}$ corresponds to an unstable region. 
$\beta_{3} / \beta_{2}$

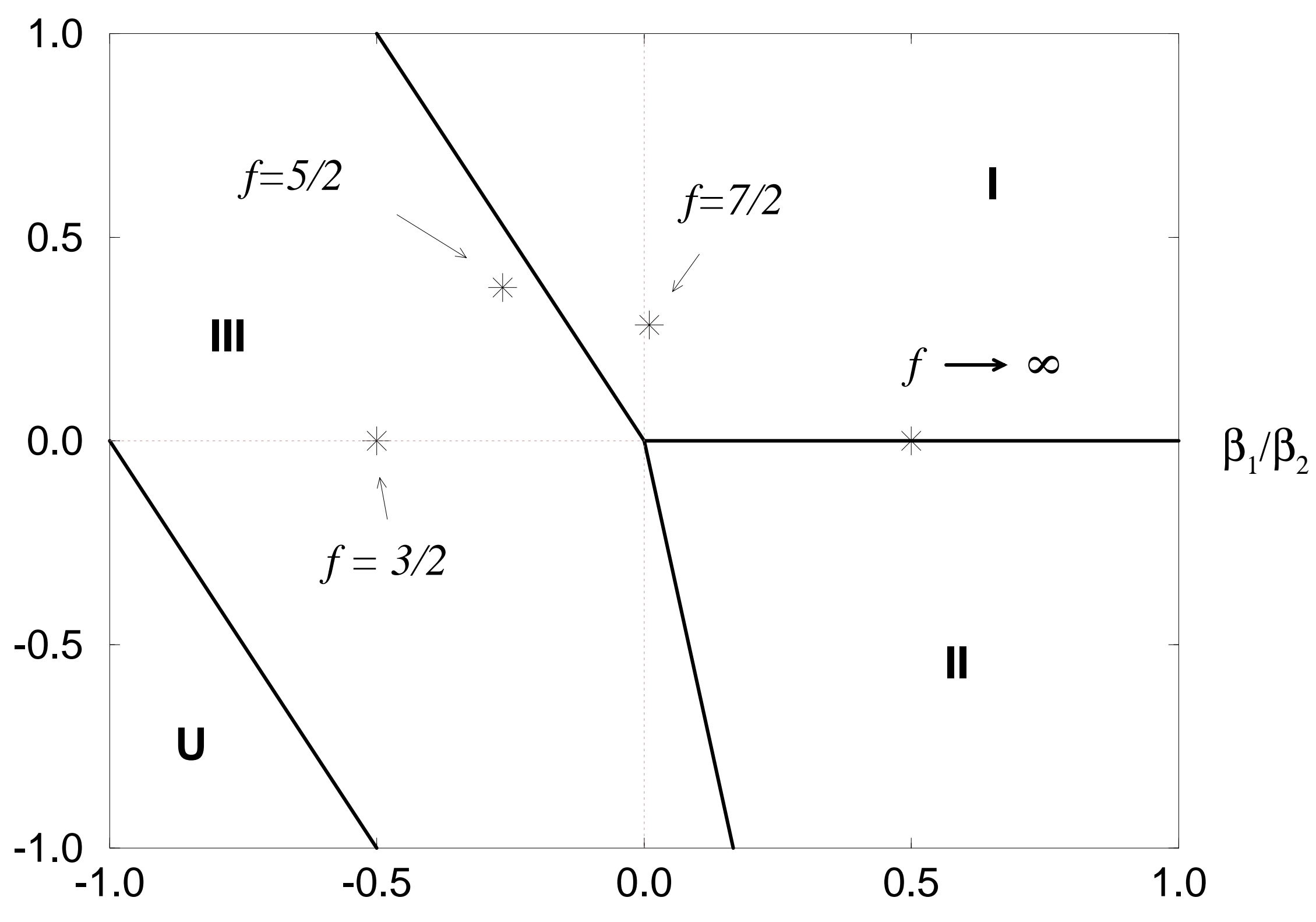

